Homology, Homotopy and Applications, vol.18(1), 2016, pp.287-294

\title{
HOMOTOPY PROPERTIES OF SOME REAL ALGEBRAIC MAPS
}

\author{
MACIEJ ZIELIŃSKI
}

(communicated by Claude Cibils)

\begin{abstract}
We generalize an old result concerning homotopy groups of regular maps between a compact real algebraic variety and a Grassmann variety to a recently introduced class of stratified-regular maps. More precisely, we show that the embedding of the space of stratified-regular maps into the space of continuous (in the Euclidean topology) maps induces an isomorphism in all homotopy groups other then zeroth and a monomorphism in the latter case.
\end{abstract}

\section{Introduction}

The aim of this paper is to generalize the main result of paper [3] to a recently introduced class of stratified-regular maps between real algebraic varieties, which is wider than the class of regular maps. This new class of maps has been studied in $[\mathbf{4}, \mathbf{6}, \mathbf{9}]$, related results can also be found in $[\mathbf{5}, \mathbf{7}, \mathbf{8}]$.

Throughout the paper by a real algebraic variety we will understand a ringed space isomorphic to an algebraic subset of $\mathbb{R}^{n}$ endowed with the Zariski topology and the sheaf of (real-valued) regular functions. Such a variety also carries the standard Euclidean topology inherited from $\mathbb{R}^{n}$ and unless explicitly stated otherwise this is the topology any topological notion we use refers to. A morphism of real algebraic varieties will be called a regular map. If $X, Y$ are real algebraic varieties then we will denote the space of all such maps by $\mathcal{R}(X, Y)$.

We will denote by $\mathbb{F}$ any of the fields $\mathbb{R}$ and $\mathbb{C}$ or the algebra $\mathbb{H}$. All $\mathbb{H}$-vector spaces are left vector spaces. If convenient we will treat $\mathbb{C}^{k}$ and $\mathbb{H}^{k}$ as real vector spaces. Then the Grassmannian $\mathbb{G}_{p}\left(\mathbb{F}^{n}\right)$ of $p$-dimensional $\mathbb{F}$-vector subspaces of $\mathbb{F}^{n}$ can be regarded as a real algebraic variety, see [1, Theorem 3.4.4].

We shall now recall the definition of stratified-regular maps given in [9]. Throughout the rest of this section $X$ and $Y$ will denote real algebraic varieties. By a stratification of $X$ we will understand a finite collection $\mathcal{X}$ of pairwise disjoint Zariski locally closed subvarieties the union of which is $X$. Each element of $\mathcal{X}$ will be called a stratum.

Definition 1.1. Let $\mathcal{X}$ be a stratification of $X$. We will say that a map $f: X \rightarrow Y$ is $\mathcal{X}$-regular if $f$ is continuous and its restriction to each stratum of $\mathcal{X}$ is a regular map. We will say that $f$ is stratified-regular if it is $\mathcal{S}$-regular for some stratification $\mathcal{S}$ of $X$. We will denote the space of all stratified-regular maps between $X$ and $Y$ by $\mathcal{R}^{0}(X, Y)$.

Received July 13, 2015, revised October 26, 2015; published on May 5, 2016. 2010 Mathematics Subject Classification: 14P05, 14P25.

Key words and phrases: algebraic vector bundle, stratified-regular map, Grassmannian. Article available at http://dx.doi.org/10.4310/HHA.2016.v18.n1.a15

Copyright (C) 2016, International Press. Permission to copy for private use granted. 
Obviously a regular map is the same as an $\{X\}$-regular map. We have inclusions $\mathcal{R}(X, Y) \hookrightarrow \mathcal{R}^{0}(X, Y) \hookrightarrow \mathcal{C}(X, Y)$, where $\mathcal{C}(X, Y)$ is the space of continuous maps. In particular we can consider $\mathcal{R}(X, Y)$ and $\mathcal{R}^{0}(X, Y)$ as topological subspaces of $\mathcal{C}(X, Y)$ equipped with the compact-open topology. The main result of paper $[\mathbf{3}]$ states that for compact $X$ and any $f \in \mathcal{R}\left(X, \mathbb{G}_{p}\left(\mathbb{F}^{n}\right)\right)$ the induced map $\pi_{k}\left(\mathcal{R}\left(X, \mathbb{G}_{p}\left(\mathbb{F}^{n}\right)\right), f\right) \rightarrow$ $\pi_{k}\left(\mathcal{C}\left(X, \mathbb{G}_{p}\left(\mathbb{F}^{n}\right)\right), f\right)$ is an isomorphism of groups for $k>0$ and is injective for $k=0$. Our aim is to get an analogous result for the space $\mathcal{R}^{0}\left(X, \mathbb{G}_{p}\left(\mathbb{F}^{n}\right)\right)$.

Theorem 1.2. Let $X$ be a compact real algebraic variety and let

$$
i: \mathcal{R}^{0}\left(X, \mathbb{G}_{p}\left(\mathbb{F}^{n}\right)\right) \hookrightarrow \mathcal{C}\left(X, \mathbb{G}_{p}\left(\mathbb{F}^{n}\right)\right)
$$

be the inclusion map. Then for any $f \in \mathcal{R}^{0}\left(X, \mathbb{G}_{p}\left(\mathbb{F}^{n}\right)\right)$ the induced map

$$
i_{*}: \pi_{k}\left(\mathcal{R}^{0}\left(X, \mathbb{G}_{p}\left(\mathbb{F}^{n}\right)\right), f\right) \rightarrow \pi_{k}\left(\mathcal{C}\left(X, \mathbb{G}_{p}\left(\mathbb{F}^{n}\right)\right), f\right)
$$

is injective for $k=0$ and a group isomorphism for $k \geqslant 1$.

There are two main ingredients of the proof. The first is the correspondence between homotopy classes of maps into the Grassmannians and vector bundles. The second is the fact that stratified-algebraic sections of stratified-algebraic bundles, which we will define following [9] in Section 2, have good approximation properties.

We shall now give some examples where the map $i_{*}: \pi_{0}\left(\mathcal{R}^{0}\left(X, \mathbb{G}_{p}\left(\mathbb{F}^{n}\right)\right), f\right) \rightarrow$ $\pi_{0}\left(\mathcal{C}\left(X, \mathbb{G}_{p}\left(\mathbb{F}^{n}\right)\right), f\right)$ is surjective and hence $i$ is a weak homotopy equivalence. Observe that this is equivalent to every continuous map from $X$ to $\mathbb{G}_{p}\left(\mathbb{F}^{n}\right)$ being homotopic to a stratified-regular one.

Example 1.3. Let $X$ be a compact real algebraic variety. If $\operatorname{dim} X \leqslant \operatorname{dim}_{\mathbb{R}} \mathbb{F}$, then $i_{*}: \pi_{k}\left(\mathcal{R}^{0}\left(X, \mathbb{G}_{p}\left(\mathbb{F}^{n}\right)\right), f\right) \rightarrow \pi_{k}\left(\mathcal{C}\left(X, \mathbb{G}_{p}\left(\mathbb{F}^{n}\right)\right), f\right)$ is surjective for any $p, n$. This follows by combining $[\mathbf{9}$, Corollary 3.6] and [9, Theorem 4.10]. In particular, this is the case when $X$ is compact of dimension $d=1,2,4$ and $\mathbb{G}_{p}\left(\mathbb{F}^{n}\right)$ a projective line of the same dimension, that is, one of $\mathbb{G}_{1}\left(\mathbb{R}^{2}\right), \mathbb{G}_{1}\left(\mathbb{C}^{2}\right)$ and $\mathbb{G}_{1}\left(\mathbb{H}^{2}\right)$ equal to, respectively, $\mathbb{S}^{1}, \mathbb{S}^{2}$ and $\mathbb{S}^{4}$. This is different from the case of regular maps as if $M$ is a smooth, closed, connected orientable surface or a boundary of a connected 5-manifold then there exists a real algebraic variety $X$ diffeomorphic to $M$ such that all regular maps from $X$ to, respectively, $\mathbb{S}^{2}, \mathbb{S}^{4}$ are null-homotopic [2, Theorems $\left.4,4.1\right]$.

Example 1.4. If $X$ is homotopy equivalent to $\mathbb{S}^{d}$ the map $i_{*}$ is surjective for any $p, n$. This can be obtained by combining [9, Corollary 4.7] and [9, Theorem 1.3]. Once again this is not true for regular maps as there are varieties diffeomorphic to the spheres $\mathbb{S}^{4 k}$ with some continuous maps to $\mathbb{G}_{p}\left(\mathbb{F}^{n}\right)$ not homotopic to regular ones [9, Example 1.4].

\section{Approximation by stratified-regular sections}

Throughout the rest of paper by a bundle we will mean an $\mathbb{F}$-vector bundle. We will denote bundles by the letters of Greek alphabet. We will denote the trivial $\mathbb{F}$-bundle of dimension $n$ over a space $X$ by $\varepsilon_{X}^{n}(\mathbb{F})$. Any notion of orthogonality we will use comes from the standard scalar products $\langle x, y\rangle=\sum_{j=1}^{n} x_{j} \bar{y}_{j}$, where $\bar{x}_{j}=x_{j}$ if $\mathbb{F}=\mathbb{R}$ and is the complex or quaternionic conjugate if $\mathbb{F}=\mathbb{C}, \mathbb{H}$, respectively.

Recall that a bundle $\xi$ on a real algebraic variety $X$ is called algebraic if it is an algebraic subbundle of $\varepsilon_{X}^{n}(\mathbb{F})$ for some $n \in \mathbb{N}$ (for equivalent formulations see 
[1, Chapter 12]). An important example of such a bundle is the tautological $\mathbb{F}$-bundle $\gamma$ on $\mathbb{G}_{p}\left(\mathbb{F}^{n}\right)$ with fiber at $x \in \mathbb{G}_{p}\left(\mathbb{F}^{n}\right)$ equal to the subspace $x \subset \mathbb{F}^{n}$ itself. We will now define stratified-algebraic bundles which have similar relationship with stratifiedregular maps as algebraic bundles with regular maps.

Definition 2.1. Let $X$ be a real algebraic variety and $\mathcal{X}$ a stratification of $X$. An $\mathbb{F}$-subbundle $\xi$ of $\varepsilon_{X}^{n}(\mathbb{F})$ will be called $\mathcal{X}$-algebraic if for every stratum $S$ of $\mathcal{X}$ the restriction $\left.\xi\right|_{S}$ is an algebraic subbundle of $\left.\varepsilon_{X}^{n}(\mathbb{F})\right|_{S}$. A section $s$ of $\xi$ will be called $\mathcal{X}$-algebraic if $\left.s\right|_{S}$ is an algebraic section of the bundle $\left.\xi\right|_{S}$ for every stratum $S$ of $\mathcal{X}$.

An $\mathbb{F}$-bundle $\eta$ will be called stratified-algebraic if it is $\mathcal{X}$-stratified for some stratification $\mathcal{X}$ and a section $r$ of $\eta$ will be called stratified-algebraic if it is $\mathcal{X}^{\prime}$-algebraic for some stratification $\mathcal{X}^{\prime}$ for which $\eta$ is $\mathcal{X}^{\prime}$-algebraic.

If $\eta$ and $\xi$ are both $\mathcal{X}$-stratified bundles on $X$ then a topological morphism of vector bundles $\phi: \xi \rightarrow \eta$ will be called $\mathcal{X}$-algebraic if for each stratum $S$ the induced morphism of algebraic $\mathbb{F}$-bundles $\left.\phi\right|_{S}:\left.\left.\xi\right|_{S} \rightarrow \eta\right|_{S}$ is algebraic.

Recall that a pullback of an algebraic bundle by a regular map is an algebraic bundle. In fact an $\mathbb{F}$-bundle of rank $p$ is algebraic iff for some $n$ it is a pullback of the tautological bundle $\gamma$ on $\mathbb{G}_{p}\left(\mathbb{F}^{n}\right)$ by a regular map - for both facts see [1, Chapter 12]. As an immediate consequence, we get that for any $\mathcal{X}$-regular map $f: X \rightarrow \mathbb{G}_{p}\left(\mathbb{F}^{n}\right)$ the pullback bundle $f^{*}(\gamma)$ is $\mathcal{X}$-algebraic.

Our aim in this section is to prove that continuous sections of a stratified-algebraic vector bundle can be approximated in the compact-open topology by sections that are stratified algebraic. We will also show that this can be done with a good degree of control. For the sake of completeness, we include relevant background results. We first recall a basic approximation lemma for regular functions on a real algebraic variety. For a continuous function $f: X \rightarrow \mathbb{R}$ and a compact subset $K \subset X$ we will write $\|f\|_{K}=\sup _{x \in K}|f(x)|$.

Lemma 2.2. [3, Lemma 2.1] Let $X$ be a real algebraic variety and $Y$ a Zariski closed subvariety of $X$. Let $K$ be a compact subset of $X$ containing $Y$. If $f: K \rightarrow \mathbb{R}$ is a continuous function whose restriction $\left.f\right|_{Y}$ is regular then for any $\varepsilon>0$ there exists a regular function $F: X \rightarrow \mathbb{R}$ such that $\|F-f\|_{K}<\varepsilon$ and $\left.F\right|_{Y}=\left.f\right|_{Y}$.

Proof. We treat $X$ as an algebraic subset of $\mathbb{R}^{k}$ for some $k \in \mathbb{N}$ with $Y$ a zero set of some polynomial $q(x), x=\left(x_{1}, \ldots, x_{k}\right) \in \mathbb{R}^{k}$. First we reduce to case where $\left.f\right|_{Y} \equiv 0$. Since $\left.f\right|_{Y}$ is regular it can be written in the form $\left.f\right|_{Y}(x)=r(x) / s(x)$ for some polynomials $r, s$ such that $s$ has no zeroes on $Y$ [1, Proposition 3.2.3]. Then the function

$$
\tilde{f}(x)=\frac{r(x) s(x)}{s(x)^{2}+q(x)^{2}}
$$

is well-defined on the whole of $X$ and regular. Moreover, $\left.\tilde{f}\right|_{Y}=\left.f\right|_{Y}$. Substituting $f-\tilde{f}$ for $f$ gives us the desired reduction.

Using the biregular embedding $X \ni x \mapsto(q(x), x) \in \mathbb{R}^{n+1}$ allows as to treat $X$ as an algebraic subset of $\mathbb{R}^{k+1}$ such that $Y=X \cap H$, where $H$ is the hyperplane given by the equation $x_{0}=0$. Note that we can then continuously extend $f$ by 0 to $K \cup H$. The Tietze extension theorem yields a continuous extension $\hat{f}: \mathbb{R}^{k+1} \rightarrow \mathbb{R}$ of $f$ such that $\left.\hat{f}\right|_{K}=f$ and $\left.\hat{f}\right|_{H} \equiv 0$. We may assume that $\hat{f}$ has compact support. 
Let $\hat{g}: \mathbb{R}^{k+1} \rightarrow \mathbb{R}$ be a smooth function with $\|\hat{g}(x)-\hat{f}(x)\|_{K}<\frac{\varepsilon}{2}$. If $\phi: \mathbb{R}^{k+1} \rightarrow$ $[0,1]$ is a smooth function with $\left.\phi\right|_{H} \equiv 1$ and support in a sufficiently thin neighborhood of $H$, then $g=(1-\phi) \hat{g}$ is smooth, satisfies $\|g-f\|_{K}<\frac{\varepsilon}{2}$ and $\left.g\right|_{H} \equiv 0$. Putting

$$
\alpha(x)=\int_{0}^{1} \frac{\partial g\left(t x_{0}, x_{1}, \ldots, x_{k}\right)}{\partial x_{0}} d t
$$

we can write $g(x)=\pi(x) \alpha(x)$, where $\pi\left(x_{0}, \ldots, x_{k}\right)=x_{0}$. Since $\alpha$ is smooth, by the Weierstrass approximation theorem there exists a polynomial $p(x)$ such that $\| \alpha-$ $p \|_{K}<\frac{\varepsilon}{2 M}$, where $M>\|\pi\|_{K}$. Putting $F(x)=\pi(x) p(x)$ we see that $F$ is regular, $\left.F\right|_{Y} \equiv 0$ and $\|F-f\|_{K} \leqslant\|\pi p-\pi \alpha\|_{K}+\|g-f\|_{K}<M \cdot \frac{\varepsilon}{2 M}+\frac{\varepsilon}{2}=\varepsilon$.

We would now like to extend Lemma 2.2 to stratified-regular functions. In order to do that we will need a result from paper $[\mathbf{6}]$ which is formulated in the language of the so-called continuous rational functions. Thus we first need to investigate the relationship between continuous rational and stratified-regular functions.

Definition 2.3. A map $f: X \rightarrow Y$ will be called continuous rational if $f$ is continuous and there exists a Zariski open and dense subvariety $Z \subset X$ such that $\left.f\right|_{Z}$ is a regular map.

A map $f: X \rightarrow Y$ will be called hereditarily rational if it is continuous and for every irreducible subvariety $S \subset X$ the restriction $\left.f\right|_{S}$ is continuous rational.

It turns out that the definitions of stratified-regular and hereditarily rational functions are equivalent. This is well known to the experts $[\mathbf{4 , 6}, \mathbf{9}]$ but we give a short proof here for the convenience of the reader.

Proposition 2.4. [6, Definition 10; 9, Proposition 2.2] Let $f: X \rightarrow Y$ be a map of real algebraic varieties. Then $f$ is hereditarily rational if and only if $f$ is stratified-regular.

Proof. Suppose $f$ is hereditarily rational. We want to find a stratification $\mathcal{X}$ of $X$ so that $f$ is $\mathcal{X}$-regular. Because $f$ is continuous rational, there exists a Zariski open and dense subvariety $X^{0} \subset X$ such that $\left.f\right|_{X^{0}}$ is regular. Since $f$ is hereditarily rational we can repeat this reasoning to the Zariski closed subvariety $X \backslash X^{0}$ and get its Zariski open and dense subvariety $X^{1}$, on which $f$ is regular. In this way we get the desired stratification $\mathcal{X}=\left\{X^{0}, X^{1}, \ldots, X^{k}\right\}$ (the process ends since the dimension of considered varieties decreases at every step of the construction).

Suppose now that $f$ is $\mathcal{X}$-regular for some stratification $\mathcal{X}$ of $X$. If $Z \subset X$ is an irreducible subvariety then the intersection $S \cap Z$ must be Zariski open and hence Zariski dense for some stratum $S$ of $\mathcal{X}$.

This allows us to apply the following result from $[\mathbf{6}]$ to the problem of approximation by stratified-regular maps.

Theorem 2.5. [6, Theorem 9, Proposition 10] Let $W \subset Z$ be real algebraic varieties and let $g: Z \rightarrow \mathbb{R}$ be a continuous rational function on $Z$ which is regular on $Z \backslash$ $W$. Then $g$ is hereditarily rational if and only if for every real algebraic variety $X$ which contains $Z$ as a closed subvariety, $g$ extends to a hereditarily rational function $G: X \rightarrow \mathbb{R}$. Moreover, $G$ can be assumed to be regular on the set $X \backslash W$.

Lemma 2.6. [9, Lemma 4.3] Let $X$ be a real algebraic variety. Let $B \subset A \subset X$ be Zariski closed subvarieties of $X$. Assume that $A$ is contained in a compact subset $K \subset X$. Let $f: X \rightarrow \mathbb{R}$ be a continuous function such that $\left.f\right|_{A}$ is stratified regular 
and $\left.f\right|_{A \backslash B}$ is regular. Then for every $\varepsilon>0$, there exists a stratified regular function $F: X \rightarrow \mathbb{R}$ such that $\left.F\right|_{A}=\left.f\right|_{A},\left.F\right|_{X \backslash B}$ is regular and $\|F-f\|_{K}<\varepsilon$.

Proof. By Proposition $\left.2.4 f\right|_{A}$ is hereditarily rational. Therefore, we can apply Theorem 2.5 to $g=\left.f\right|_{A}$ with $Z=A$ and $W=B$. Then we get an extension $\tilde{f}: X \rightarrow \mathbb{R}$ which is regular in restriction to $X \backslash B$ and stratified regular. The function $f-\tilde{f}$ is continuous and zero on $A$. By Lemma 2.2 there exists a regular function $\psi: X \rightarrow \mathbb{R}$ such that $\left.\psi\right|_{A} \equiv 0$ and $\|f-\tilde{f}-\psi\|_{K}<\varepsilon$. We can then put $F=\tilde{f}+\psi$.

The final lemma of this section is an application of Lemma 2.6 to the approximation of continuous sections of stratified-algebraic bundles. If $\mathcal{X}$ is a stratification of a variety $X$, we will, for a Zariski closed subvariety $Y \subset X$, denote by $\mathcal{X} \cap Y$ the induced stratification $\{S \cap Y \mid S \in \mathcal{X}\}$.

Lemma 2.7. Let $X$ be a real algebraic variety and $Y$ a Zariski closed subvariety contained in a compact subset $K \subset X$. Let $\mathcal{X}$ be a stratification of $X$ and let $\xi$ be an $\mathcal{X}$-algebraic $\mathbb{F}$-bundle of constant dimension on $X$. Suppose that $\sigma: K \rightarrow \xi$ is a continuous section of $\xi$ whose restriction $\left.\sigma\right|_{Y}$ is $\mathcal{X} \cap Y$-algebraic. Let $\mathcal{U}$ be a neighborhood of $\sigma$ in the space of all continuous sections of $\xi$ equipped with the compact-open topology. Then there exists an $\mathcal{X}$-algebraic section $s$ of $\xi$ such that $\left.s\right|_{Y}=\left.\sigma\right|_{Y}$ and $\left.s\right|_{K} \in \mathcal{U}$.

Proof. Since $\xi$ is a subbundle of some trivial bundle $\varepsilon=\varepsilon_{X}^{n}(\mathbb{F})$, there exists a complementary orthogonal bundle $\xi^{\perp}$ such that $\xi \oplus \xi^{\perp}=\varepsilon_{Y}^{n}(\mathbb{F})$. Then the isomorphism of restrictions $\left.\left.\xi\right|_{S} \oplus \xi^{\perp}\right|_{S}=\left.\varepsilon\right|_{S}$ is algebraic for any stratum $S \in \mathcal{X}$ (see the characterization of complementary algebraic bundles in [1, Theorem 12.1.7]) and the projection $P:\left.\left.\varepsilon\right|_{S} \rightarrow \xi\right|_{S}$ is a morphism of algebraic bundles.

We can now reduce the problem to $\xi=\varepsilon$, for if $\tilde{s}$ is an $\mathcal{X}$-algebraic section of $\varepsilon$ approximating $\sigma$, then $P \circ \tilde{s}$ is an $\mathcal{X}$-algebraic section of $\xi$ also approximating $\sigma$. Regarding $\sigma$ as a map into $\mathbb{F}^{n}$ we can approximate its component functions using Lemma 2.6. It suffices now to observe that, since they are regular on a complement of a subset of $Y$, the approximating functions can be indeed regarded as $\mathcal{X}$-algebraic.

\section{Proof of the main theorem}

We will now give two preparatory lemmas which apply the general results of the previous section to the particular situation of Theorem 1.2. Since that will involve a lot of work with maps defined on products of spaces, we will introduce a notation which shortens the statements and proofs. From now on for a map $f: X \times Y \rightarrow Z$ and a subset $A \subset Y$ we will denote by $f_{A}$ the restriction $\left.f\right|_{X \times A}$. If $A=\{y\}$ we will omit the brackets and write $f_{y}$. For a stratification $\mathcal{X}$ of a variety $X$ and a variety $Y$ we will denote by $\mathcal{X} \times Y$ the stratification $\{S \times Y \mid S \in \mathcal{X}\}$ of $X \times Y$.

Lemma 3.1. Let $X$ and $Y$ be real algebraic varieties and let $\mathcal{X}$ be a stratification of $X$. Let $Y_{0}, Y_{1} \subset Y$ be Zariski closed algebraic subvarieties with $Y_{0}$ nonempty. Assume that $X$ is compact and that there exists a compact, contractible subset $K$ of $Y$ such that $Y_{0} \cup Y_{1} \subset K$. Let $F: X \times K \rightarrow \mathbb{G}_{p}\left(\mathbb{F}^{n}\right)$ be a continuous map whose restriction $F_{Y_{0}}=$ $\left.F\right|_{X \times Y_{0}}$ is $\mathcal{X} \times Y_{0}$-regular. Then for any neighborhood $\mathcal{U}$ of $F$ in $\mathcal{C}\left(X \times K, \mathbb{G}_{p}\left(\mathbb{F}^{n}\right)\right)$ there exists a map $G \in \mathcal{U}$ such that $G_{Y_{0}}=F_{Y_{0}}, G_{Y_{1}}$ is $\mathcal{X} \times Y_{1}$-regular and $G_{y}$ is $\mathcal{X} \times\{y\}$-regular for all $y \in K$. 
Proof. The idea is to use the connection between maps into Grassmannians and bundles. We will "change" the map $F$ into a pullback bundle and then use the approximation Lemma 2.7 to get a stratified-algebraic bundle which is "close" and can be used to obtain the approximating map.

Let $\gamma$ be the tautological $\mathbb{F}$-bundle on $\mathbb{G}_{p}\left(\mathbb{F}^{n}\right)$. Pick a point $y_{0} \in Y_{0}$ and let $f: X \times$ $Y \rightarrow \mathbb{G}_{p}\left(\mathbb{F}^{n}\right)$ be the map given by $f(x, y)=F\left(x, y_{0}\right)$. Consider the pullback $\mathbb{F}$-bundles $\xi=f^{*}(\gamma)$ on $X \times Y$ and $\eta=F_{K}^{*}(\gamma)$ on $X \times K$. Since $f$ is clearly $\mathcal{X} \times Y$-regular, the bundle $\xi$ is $\mathcal{X} \times Y$-algebraic as a pullback of an algebraic bundle by an $\mathcal{X} \times Y$-regular map. For the same reason the bundle $\left.\eta\right|_{X \times Y_{0}}$ is $\mathcal{X} \times Y_{0}$-algebraic.

A contraction of a contractible set $K$ to the point $y_{0}$ induces a homotopy between the maps $F_{K}$ and $f_{K}$. Hence the bundles $\left.\xi\right|_{X \times K}$ and $\eta$ are topologically isomorphic. Let $\phi:\left.\xi\right|_{X \times K} \rightarrow \eta$ be a topological $\mathbb{F}$-bundle isomorphism. Let $\alpha$ be the continuous section of the bundle $\operatorname{Hom}\left(\left.\xi\right|_{\mathrm{X} \times \mathrm{K}}, \eta\right)$ induced by $\phi$, that is, $\alpha(x, y)(v)=\phi(v)$ for any $v$ in the fiber $\xi_{(x, y)}$ of $\xi$ at the point $(x, y)$. Since the bundles $\left.\xi\right|_{X \times Y_{0}}$ and $\left.\eta\right|_{X \times Y_{0}}$ are $\mathcal{X} \times$ $Y_{0}$-algebraic, so is the bundle $\operatorname{Hom}\left(\left.\xi\right|_{\mathrm{X} \times \mathrm{Y}_{0}},\left.\eta\right|_{\mathrm{X} \times \mathrm{Y}_{0}}\right)=\left.\operatorname{Hom}(\xi, \eta)\right|_{\mathrm{X} \times \mathrm{Y}_{0}}$. Therefore, by Lemma 2.7 there exists an $\mathcal{X} \times Y_{0}$-algebraic section $\alpha_{0}: X \times Y_{0} \rightarrow \operatorname{Hom}(\xi, \eta) \mid \mathrm{X} \times \mathrm{Y}_{0}$ arbitrarily close to $\left.\alpha\right|_{X \times Y_{0}}$.

The continuous section $\alpha_{0}$ is defined on a closed subset of $X \times K$ and can, therefore, be extended to a continuous section $\beta_{0}$ on $X \times K$. Then if $\tau: X \times K \rightarrow[0,1]$ is a continuous function which is 1 on $X \times Y_{0}$ and has support in a sufficiently small neighborhood of $X \times Y_{0}$, the section $\beta=\tau \beta_{0}+(1-\tau) \alpha$ is a continuous extension of $\alpha_{0}$ arbitrarily close to $\alpha$. Since the set of fiberwise linear isomorphisms is open, we can assume that $\beta$ is an isomorphism on fibers.

Recall that there is an algebraic splitting $\varepsilon_{\mathbb{G}_{p}\left(\mathbb{F}^{n}\right)}^{n}(\mathbb{F})=\gamma \oplus \gamma^{\perp}$ (see $[\mathbf{1}$, Proposition 12.1.8]). Hence $\eta=F_{K}^{*}(\gamma)$ can be regarded as a subbundle of $\left.\varepsilon\right|_{X \times K}$, where $\varepsilon$ is the trivial bundle $\varepsilon_{X \times Y}^{n}(\mathbb{F})$. This allows us to treat $\beta$ as a section of $\operatorname{Hom}(\xi, \varepsilon)$ defined on $X \times K$. Since the bundle $\operatorname{Hom}(\xi, \varepsilon)$ is clearly $\mathcal{X} \times Y$-algebraic, Lemma 2.7 yields an $\mathcal{X} \times Y$-algebraic section $s: X \times Y \rightarrow \operatorname{Hom}(\xi, \varepsilon)$ such that $\left.s\right|_{X \times K}$ is arbitrarily close to $\beta$ and $s$ agrees with $\beta$ on $X \times Y_{0}$.

By treating $\eta$ as a subbundle of $\varepsilon$ we can identify its fibers with subspaces of $\mathbb{F}^{n}$, that is, the points of $\mathbb{G}_{p}\left(\mathbb{F}^{n}\right)$. Then we have $\beta(x, y)\left(\xi_{(x, y)}\right)=\eta_{(x, y)}=\gamma_{F(x, y)}=F(x, y)$ for any $(x, y)$ in $X \times K$. We can make $\left.s\right|_{X \times K}$ a fiberwise linear monomorphism by choosing it close enough to $\beta$. Then the map $G: X \times K \rightarrow \mathbb{G}_{p}\left(\mathbb{F}^{n}\right)$ given by $G(x, y)=$ $s(x, y)\left(\xi_{(x, y)}\right)$ is well-defined. We see that $G(x, y)$ is then close to $\eta_{(x, y)}=F(x, y)$ and because $s$ agrees with $\beta$ on $X \times Y_{0}$ we have an equality there. Moreover, $s$ was $\mathcal{X} \times Y$ algebraic, so clearly $G_{y}$ is also $\mathcal{X} \times\{y\}$-algebraic for any $y$ in $K$.

Lemma 3.2. Let $X$ be a compact real algebraic variety, and $F: X \times \mathbb{S}^{k} \rightarrow \mathbb{G}_{p}\left(\mathbb{F}^{n}\right)$ a continuous map. Suppose there exists a point $y_{0} \in \mathbb{S}^{k}$ and a stratification $\mathcal{X}$ of $X$ such that the map $F_{y_{0}}$ is $\mathcal{X} \times\left\{y_{0}\right\}$-regular. Let $\mathcal{U}$ be a neighborhood of $F$ in $\mathcal{C}(X \times$ $\left.\mathbb{S}^{k}, \mathbb{G}_{p}\left(\mathbb{F}^{n}\right)\right)$. Then there exists a map $G \in \mathcal{U}$ such that $G_{y_{0}}=F_{y_{0}}$ and $G_{y}$ is $\mathcal{X} \times\{y\}$ regular for all $y$ in $\mathbb{S}^{k}$.

Proof. Let $H_{1}$ and $H_{2}$ be the closed hemispheres in $\mathbb{S}^{k}$ intersecting in the equator $(k-1)$-sphere $E$. Without loss of generality assume $y_{0} \in E$. Applying Lemma 3.1 to $Y=\mathbb{S}^{k}, K=H_{1}, Y_{0}=\left\{y_{0}\right\}$ and $Y_{1}=E$ yields a continuous map $G^{0}: X \times H_{1} \rightarrow$ $\mathbb{G}_{p}\left(\mathbb{F}^{n}\right)$, which is arbitrarily close to $F_{H_{1}}$, such that $G_{y_{0}}^{0}=F_{y_{0}}$ and $G_{y}^{0}$ is $\mathcal{X} \times\{y\}$ regular for all $y$ in $H_{1}$. 
Now, we can construct a continuous extension $G^{1}$ of $G^{0}$ to the whole of $X \times \mathbb{S}^{k}$ which is close to $F$. This can be done using a tubular neighborhood of an embedded Grassmannian and an argument similar as in the proof of the Whitney Approximation Theorem [10, Theorem 10.16]. We then apply Lemma 3.1 once more, this time to $F=G_{H_{2}}^{1}$ with $K=H_{2}, Y_{0}=E$ and $Y_{1}=\emptyset$. This gives us a continuous map $G^{2}: X \times$ $H_{2} \rightarrow \mathbb{G}_{p}\left(\mathbb{F}^{n}\right)$ such that $G_{E}^{2}=G_{E}^{1}$ and $G_{y}$ is $X \times\{y\}$-regular for all $y$ in $H_{2}$. Since $G^{1}$ and $G^{2}$ agree on $E$ they can be glued together to a continuous map $G$ defined on $X \times \mathbb{S}^{k}$. It is clear that, since $G^{i}$ can be chosen arbitrarily close to $\left.F\right|_{H_{i}}, G$ can be chosen in such a way that it belongs to $\mathcal{U}$ and has all the required properties.

We are now ready to prove the main theorem. It is worth observing that the main result of $[\mathbf{3}]$ implies the surjectivity of the map $i_{*}$ for $k>0$ as it shows that $i_{*} \circ j_{*}$ is surjective, where $j_{*}: \pi_{k}\left(\mathcal{R}\left(X, \mathbb{G}_{p}\left(\mathbb{F}^{n}\right)\right), f\right) \rightarrow \pi_{k}\left(\mathcal{R}^{0}\left(X, \mathbb{G}_{p}\left(\mathbb{F}^{n}\right)\right), f\right)$ is the map induced by the embedding. Nevertheless, we will also prove the surjectivity as the argument is basically the same as in $[\mathbf{3}]$.

To make the proof clearer we will identify any map $g: \mathbb{S}^{k} \rightarrow \mathcal{R}^{0}\left(X, \mathbb{G}_{p}\left(\mathbb{F}^{n}\right)\right)$ and the induced map $\tilde{g}: X \times \mathbb{S}^{k} \rightarrow \mathbb{G}_{p}\left(\mathbb{F}^{n}\right)$ given by $X \times \mathbb{S}^{k} \ni(x, y) \mapsto g(y)(x)$. Conversely, if $\mathcal{X}$ is a stratification of $X$ and the restriction $h_{y}$ of a continuous map $h: X \times \mathbb{S}^{k} \rightarrow$ $\mathbb{G}_{p}\left(\mathbb{F}^{n}\right)$ is $\mathcal{X}$-regular for every $y \in \mathbb{S}^{k}$, we will identify $h$ with the corresponding map $\mathbb{S}^{k} \rightarrow \mathcal{R}^{0}\left(X, \mathbb{G}_{p}\left(\mathbb{F}^{n}\right)\right)$. We will do the same for maps from $\mathbb{S}^{k}$ into $\mathcal{C}\left(X, \mathbb{G}_{p}\left(\mathbb{F}^{n}\right)\right)$.

Proof of Theorem 1.2. We will first show the injectivity of $i_{*}$ for all $k \geqslant 0$. Let $x_{0} \in \mathbb{S}^{k}$ and $f \in \mathcal{R}^{0}\left(X, \mathbb{G}_{p}\left(\mathbb{F}^{n}\right)\right)$ be the basepoints with $f \mathcal{X}$-regular for some stratification $\mathcal{X}$ of $X$. Suppose that $i_{*}([\phi])=0$ for some class $[\phi] \in \pi_{k}\left(\mathcal{R}^{0}\left(X, \mathbb{G}_{p}\left(\mathbb{F}^{n}\right)\right), f\right)$. This means that the map $\phi: X \times \mathbb{S}^{k} \rightarrow \mathbb{G}_{p}\left(\mathbb{F}^{n}\right)$ can be extended to a continuous map $\Phi: X \times$ $D^{k+1} \rightarrow \mathbb{G}_{p}\left(\mathbb{F}^{n}\right)$ such that $\Phi_{\mathbb{S}^{k}}=\phi$, where $D^{k+1}$ is the $(k+1)$-disk bounded by $\mathbb{S}^{k}$. Since $\Phi_{y_{0}}=f$ is $\mathcal{X} \times\left\{y_{0}\right\}$-regular, we can apply Lemma 3.1 to $\Phi$ with $Y=\mathbb{R}^{k+1}, K=$ $D^{k+1}, Y_{0}=\left\{y_{0}\right\}$ and $Y_{1}=\emptyset$ and thus get a continuous map $\tilde{\Phi}: X \times D^{k+1} \rightarrow \mathbb{G}_{p}\left(\mathbb{F}^{n}\right)$, which is arbitrarily close to $\Phi$, such that $\tilde{\Phi}_{y_{0}}=\Phi_{y_{0}}$ and $\tilde{\Phi}_{y}$ is $\mathcal{X} \times\{y\}$-regular for all $y$ in $D^{k+1}$. Since $\tilde{\phi}=\tilde{\Phi}_{\mathbb{S}^{k}}$ is null-homotopic as a map $\tilde{\phi}: \mathbb{S}^{k} \rightarrow \mathcal{R}^{0}\left(X, \mathbb{G}_{p}\left(\mathbb{F}^{n}\right)\right)$, the proof will be over if we show that there is a homotopy $H: \mathbb{S}^{k} \times[0,1] \rightarrow \mathcal{R}^{0}\left(X, \mathbb{G}_{p}\left(\mathbb{F}^{n}\right)\right)$ between the maps $\phi$ and $\tilde{\phi}$, as it will mean that $[\phi]=[\tilde{\phi}]$ in $\pi_{k}\left(\mathcal{R}^{0}\left(X, \mathbb{G}_{p}\left(\mathbb{F}^{n}\right)\right), f\right)$.

We now construct the homotopy. Recall that $\mathbb{G}_{p}\left(\mathbb{F}^{n}\right)$ with its structure of a real algebraic variety can be identified with a subset of the space of $n$ by $n$ matrices with entries in $\mathbb{F}$. Fixing a scalar (hermitian if $\mathbb{F}=\mathbb{C}, \mathbb{H}$ ) product and an orthonormal basis, we identify a subspace $V \subset \mathbb{F}^{n}$ with the matrix $A_{V}$ of the orthogonal projection onto $V$ (for details see [1, Theorem 3.4.4]). Consider the Zariski open subset $U=\left\{\left(V, V^{\prime}\right) \in\right.$ $\left.\mathbb{G}_{p}\left(\mathbb{F}^{n}\right) \times \mathbb{G}_{p}\left(\mathbb{F}^{n}\right) \mid V^{\prime} \cap V^{\perp}=\{0\}\right\}$, where $V^{\perp}$ is the orthogonal complement and the map $P: U \times[0,1] \rightarrow \mathbb{G}_{p}\left(\mathbb{F}^{n}\right)$ given by

$$
P\left(V, V^{\prime}, t\right)=\left\{x+t y \mid x \in V, y \in V^{\perp}, x+y \in V^{\prime}\right\} .
$$

Observe that, using the identification discussed above, $P_{t}\left(V, V^{\perp}\right)$ is the matrix of the projection onto the column space of the matrix:

$$
M=\left(A_{V}+t A_{V^{\perp}}\right) A_{V^{\prime}}=\left(A_{V}+t\left(\mathbb{I}_{n}-A_{V}\right)\right) A_{V^{\prime}}=t A_{V^{\prime}}+(1-t) A_{V} A_{V^{\prime}} .
$$

By the known formula from linear algebra if $B$ is the matrix consisting of $p$ linearly independent columns of $M$, then $P\left(V, V^{\prime}, t\right)=B\left(B^{*} B\right)^{-1} B^{*}$. Since the set where given $p$ columns of $M$ are linearly independent is Zariski open, we see that $P_{t}$ is regular as it can be locally expressed using regular functions. Choosing $\tilde{\phi}$ close enough 
to $\phi$ we can assume that $(\phi(x, y), \tilde{\phi}(x, y)) \in U$ for all $(x, y) \in X \times \mathbb{S}^{k}$. Then the map $H(x, y, t)=P(\phi(x, y), \tilde{\phi}(x, y), t)$ is a homotopy between $\phi$ and $\tilde{\phi}$ and, because for fixed $y$ and $t$ the map $X \ni x \mapsto H(x, y, t)$ is $\mathcal{X}$-regular as a composition of a regular map with an $\mathcal{X}$-regular map, it takes values in $\mathcal{R}^{0}\left(X, \mathbb{G}_{p}\left(\mathbb{F}^{n}\right)\right)$. This ends the proof of injectivity.

Now we prove that the map $i_{*}$ is surjective. Let $\phi: \mathbb{S}^{k} \rightarrow \mathcal{C}\left(X, \mathbb{G}_{p}\left(\mathbb{F}^{n}\right)\right)$ be a continuous map with $\phi\left(y_{0}\right)=f$. We need to construct a basepoint-preserving homotopy between $\phi$ and some map $\phi: \mathbb{S}^{k} \rightarrow \mathcal{R}^{0}\left(X, \mathbb{G}_{p}\left(\mathbb{F}^{n}\right)\right)$ which also maps $y_{0}$ to $f$. Consider a tubular neighborhood $U$ of $\mathbb{G}_{p}\left(\mathbb{F}^{n}\right)$ in some $\mathbb{R}^{N}$ and a retraction $r: U \rightarrow G$. If we assume that $t \phi+(1-t) \tilde{\phi}$ maps $X \times \mathbb{S}^{k}$ to $U$, which will happen if $\tilde{\phi}$ is close enough to $\phi$, then $H(x, y, t)=r(t \phi(x, y)+(1-t) \tilde{\phi}(x, y))$ will be the desired homotopy. It is now enough to observe that by Lemma $3.2 \tilde{\phi}$ satisfying the necessary conditions can be found arbitrarily close to $\phi$.

\section{References}

[1] J. Bochnak, M. Coste, and M.-F. Roy, Real Algebraic Geometry, Ergeb. Math. Grenzgeb. (3), vol. 36, Springer-Verlag, Berlin, 1998.

[2] J. Bochnak and W. Kucharz, On real algebraic morphisms into even-dimensional spheres, Ann. Math. (2) 128 (1988), no. 2, 415-433.

[3] London Math. Soc. 25 (1993), no. 4, 385-392.

[4] G. Fichou, J. Huisman, F. Mangolte, and J.-Ph. Monnier, Fonctions régulues (2012), to appear in J. Reine Angew. Math. doi:10.1515/crelle-2014-0034, available at arXiv:1112.3800 [math. AG].

[5] G. Fichou, J.-Ph. Monnier, and R. Quarez, Continuous functions in the plane regular after one blowing-up (2014), available at arXiv:1409.8223 [math. AG].

[6] J. Kollár and K. Nowak, Continuous rational functions on real and p-adic varieties, Math. Z. 279 (2015), no. 1-2, 85-97.

[7] W. Kucharz, Rational maps in real algebraic geometry, Adv. Geom. 9 (2009), no. $4,517-539$.

[8] W Kucharz, Approximation by continuous rational maps into spheres, J. Eur. Math. Soc. (JEMS) 16 (2014), no. 8, 1555-1569.

[9] W. Kucharz and K. Kurdyka, Stratified-algebraic vector bundles (2013), to appear in J. Reine Angew. Math. doi:10.1515/crelle-2015-0105, available at arXiv1308. 4376 [math. AG] .

[10] John M. Lee, Introduction to smooth manifolds, Grad. Texts in Math., vol. 218, Springer, New York, 2013.

Maciej Zieliński maciej.zielinski@im.uj.edu.pl

Institute of Mathematics, Faculty of Mathematics and Computer Science, Jagiellonian University, ul. Łojasiewicza 6, 30-348 Kraków, Poland 